\title{
The pentavalent antimonial therapy against experimental Leishmania amazonensis infection is more effective under the inhibition of the NF-kB pathway
}

\author{
Sharon Rose Aragão Macedo a,b,1 , Larissa Deadame de Figueiredo Nicolete ${ }^{\mathrm{a}, \mathrm{b}, 1}$, Amália dos Santos Ferreira ${ }^{\mathrm{a}}$, \\ Neuza Biguinati de Barros ${ }^{\mathrm{a}, \mathrm{c}}$, Roberto Nicolete ${ }^{\mathrm{a}, \mathrm{b}, \mathrm{c}, *}$
}

a Laboratório de Biotecnologia Aplicada à Saúde, Fundação Oswaldo Cruz (Fiocruz Rondônia), BR-364, Km 3,5, 76812-245 Porto Velho, RO, Brazil

b Programa de Pós-Graduação em Biologia Experimental (PGBioexp), Universidade Federal de Rondônia, BR-364, Km 9,5, 76801-059 Porto Velho, RO, Brazil

c Programa de Pós-Graduação em Biodiversidade e Biotecnologia da Amazônia Legal (Bionorte), BR-364, Km 5,5, 76815-800 Porto Velho, RO, Brazil

\section{A R T I C L E I N F O}

\section{Article history:}

Received 10 March 2015

Received in revised form 6 July 2015

Accepted 7 July 2015

Available online 25 July 2015

\section{Keywords:}

Leishmania amazonensis

Antimonial therapy

TNF- $\alpha$ inhibition

IL-1ß

Nitric oxide

Immune response

\begin{abstract}
A B S T R A C T
During Leishmania infection, host immune response is important to prevent the growth/survival of intracellular amastigotes. In this study, we evaluated in vitro and in vivo whether or not during Leishmania amazonensis infection, pentavalent antimonial treatment/therapy could be more effective under TNF- $\alpha$ inhibition. Both L. amazonensis-infected macrophages (in vitro model) and mice (in vivo model) were treated with a nuclear factor- $\kappa B(N F-\kappa B)$ inhibitor and with Glucantime ${ }^{\circledR}$, alone and in combined administrations. The in vitro amastigote counts, cytokines and nitrites' production were assessed after $48 \mathrm{~h}$ incubation with the drugs. Paw lesion sizes and amastigote counts were also evaluated in vivo. Quantification of IL-1ß from the infected tissue was performed. In vitro results show that when infected macrophages were incubated with QNZ + Glucantime ${ }^{\circledR}$, a greater clearance was observed for the amastigotes' growth and this was related to greater nitrite production compared to the group that was only infected. In vivo results show that mice that received the combined treatment had their paw lesion sizes and amastigote nests inside the macrophages greatly diminished, correlating with increased IL-1ß levels.
\end{abstract}

(C) 2015 Elsevier B.V. All rights reserved.

\section{Introduction}

Leishmaniasis is a disease caused by protozoan parasites from the genus Leishmania and occurs in three clinical forms: cutaneous (CL), mucocutaneous (MCL) and visceral leishmaniasis (VL). Since 1945, the first choice of treatment for cutaneous leishmaniasis has been restricted to the use of tri and pentavalent antimonials and in some cases, amphotericin B and pentamidine are also used as second and third choices, respectively. These drugs are difficult to administer, some of them are expensive and are becoming ineffective in several endemic regions. In addition, drugs used in the chemotherapy of leishmaniasis have several side effects, treatment failure and cases of relapse [1]. Because of this, the host immune response is fundamental to solve the infection. In this context, during Leishmania infection, cytokines and other mediators produced by the $\mathrm{T}_{\mathrm{H}} 1$-protective response are important to prevent the growth/survival of intracellular parasites (amastigote forms) and control their dissemination to other tissues and organs. The success of infection in

* Corresponding author at: Fiocruz Rondônia: Rua da Beira, 7671, BR364, Km 3,5, Lagoa, 76812-245, Porto Velho, RO, Brazil.

E-mail address: rnicolete@gmail.com (R. Nicolete).

1 These two authors contributed equally to this work. macrophages depends on the parasite's ability to adhere to and be phagocytosed by these cells, escaping microbicidal mechanisms [2].

Cytokines produced by the $\mathrm{T}_{\mathrm{H}} 1$-protective response, such as IFN- $\gamma$, TNF$\alpha$, and IL-1 $1 \beta$ are able to control the infection [3] while those produced by $\mathrm{T}_{\mathrm{H}} 2$ clones, such as IL- 4 , TGF- $\beta$ and IL-10 lead to the progression of the disease [4-6]. In the context of Leishmania infection, after the parasites' phagocytosis, macrophages are activated to produce microbicidal agents, which are highly toxic to the intracellular amastigotes [7]. In response to microbial stimulation, especially in the course of Leishmania infection, macrophages are able to differentiate into M1 and M2 phenotypes [8].

Studies conducted with experimental models and humans have shown that Leishmania amazonensis is able to modulate the host immune response and sustain the infection, further inhibiting antimicrobial molecule production by macrophages [9].

The CL pathology and parasite replication, along with tissue destruction and scarring are the major concerns for proposing new therapies. Cytokines and other cell signaling molecules, such as nitric oxide (NO) are able to influence the course of infection in a complex manner. In this regard, effector cytokines such as TNF- $\alpha$, IL-12 and IFN- $\gamma$, which control parasite replication may also induce tissue damage [10]. Therapies targeting these aspects of immunological mediators and their implications are emerging. The importance of TNF- $\alpha$ production by host 
cells during Leishmania infection has been discussed in several articles, including evidences of a possible healing process with pharmacological inhibitors as an alternative for refractories to conventional treatments [11-13]. The nuclear factor-kB (NF-kB) pathway is important for APCs during different extracellular stimuli to lead to the production of inflammatory cytokines and other immune mediators for controlling the infection.

In the present study we investigated whether the conventional pentavalent antimonial therapy with Meglumine antimonate (Glucantime ${ }^{\circledR}$ ) combined with a potent TNF- $\alpha$ blocking agent - NF- $\kappa$ B inhibitor - QNZ (N4-[2-(4-phenoxyphenyl) ethyl]) could lead to a different protective profile elicited by cellular immune response during in vivo L. amazonensis infection.

\section{Materials and methods}

\subsection{Animals}

BALB/c mice aged 15 to 20 weeks, weighing between 18 and $20 \mathrm{~g}$ were obtained from the vivarium of FIOCRUZ-RO and maintained under conditions of free access to water and food, respecting the $12 \mathrm{~h}$ light-dark cycle. All experiments were performed in accordance with standards established by the Ethics Committee of FIOCRUZ-RO, Brazil for the use of experimental animals (approved CEUA Protocol 2014/19).

\subsection{In vitro infection of peritoneal macrophages}

BALB/c mice were i.p. inoculated with $2 \mathrm{~mL}$ of $3 \%$ thioglycolate (Sigma-Aldrich). After 5 days, the peritoneal fluid with macrophages was recovered and plated as previously described [14]. The cells $\left(5 \times 10^{5} /\right.$ well $)$ were plated in a 24 well plate containing sterile coverslips and incubated at $37{ }^{\circ} \mathrm{C}$ in a humidified atmosphere containing $5 \% \mathrm{CO}_{2}$. After $24 \mathrm{~h}$ incubation, macrophages were washed twice with RPMI medium and then infected with $25 \times 10^{5}$ promastigotes of $L$. amazonensis (IFLA/BR/67/PH8) at $33{ }^{\circ} \mathrm{C}$ for $24 \mathrm{~h}$. After that period, the infected macrophages were washed with RPMI and incubated for $48 \mathrm{~h}$ with $10 \mu \mathrm{M}$ of QNZ (Cayman Chemical) - G2 group, $300 \mu \mathrm{g} / \mathrm{mL}$ of Glucantime ${ }^{\circledR}$ (Aventis) --G3 group and $10 \mu \mathrm{M}$ of $\mathrm{QNZ}+300 \mu \mathrm{g} / \mathrm{mL}$ of Glucantime ${ }^{\circledR}-\mathrm{G} 4$ group. Then, the culture supernatants were collected and frozen for further nitrite and cytokine quantification. The number of intracellular parasites (amastigotes) was assessed by counting 100 macrophages in bright field microscopy (1000x magnification) after panoptic staining (Instant-Prov, Pinhais, Paraná, Brasil).

\subsection{Quantification of cytokines}

The cytokines IL-1 $1 \beta$, IL-10 and IL-12 were measured in the supernatants of cultures of peritoneal macrophages collected from the assay described above. For the in vivo assay, the supernatants for IL-1 $\beta$ measurements were obtained from the maceration of paw-infected tissue (per $\mathrm{mg}$ ) after an excision procedure. Cytokine levels were quantified as $\mathrm{pg} / \mathrm{mL}$ by ELISA (BD OptEIA ${ }^{\mathrm{TM}}$, BD Biosciences), according to the manufacturer's instructions.

\subsection{NO production by macrophages}

Nitrite production by infected macrophages was determined by the Griess reaction. Supernatants $(0.1 \mathrm{ml})$ were incubated with an equal volume of Griess reagent mixtures ( $1 \%$ sulfanilamine, $0.1 \% \mathrm{~N}-(1-$ naphtyl)-ethylendiamine dihydrochloride, $2.5 \% \mathrm{H}_{3} \mathrm{PO}_{4}$ ) at room temperature for $10 \mathrm{~min}$. The absorbance was measured in a microplate reader at $540 \mathrm{~nm}$ and concentrations calculated from a sodium nitrite standard curve. Data are presented as micromoles of $\mathrm{NO}_{2}^{-}$(nitrite).

\subsection{In vivo infection and treatments}

$\mathrm{BALB} / \mathrm{c}$ mice were inoculated in the right hind paw with $10^{5}$ promastigotes of L. amazonensis [14]. Eight weeks after the infection mice were separated according to the treatment received in four groups. During five consecutive days each group was i.p. injected with $300 \mu \mathrm{L}$ as follow: G1 - Control Group that received PBS 1×; G2 - QNZ Group $0.001 \mathrm{~g} / \mathrm{kg}$ of QNZ only; G3 - Glucantime ${ }^{\circledR}$ group - $0.1 \mathrm{~g} / \mathrm{kg}$; G4 QNZ + Glucantime ${ }^{\circledR}$ group - association of the concentrations above, equal volume injected in the same preparation. The progression of the lesion was monitored accordingly [14], briefly, at the beginning and the end of the treatment the size of the lesions was monitored with a caliper. The infected animals were evaluated by comparing their paws' lesion sizes to the measurement of the uninfected contralateral paw (CP).

\subsection{Histological analysis of the infected paws}

Mice paws were collected, fixed and stored in 10\% formalin. Subsequently, they were decalcified in EDTA solution at 10\% in PBS for 4 days at a temperature of $4{ }^{\circ} \mathrm{C}$. The samples were then subjected to a paraffin embedding process for sagittal section $(5 \mu \mathrm{m})$. The sections were dehydrated and diaphanized using increasing concentrations of alcohols and xylol, stained with hematoxylin and eosin and covered with coverslips for further analysis in light microscopy at 200 and $400 \times$ magnification to assess the intensity of amastigotes in the tissue [14]. For each amastigote nest in the tissue $(n=4)$, the counted particles ( 2 to $3.5 \mu \mathrm{m}$ ) as amastigote-like were determined in 5 to 6 fields using ImageJ software (NIH).

\subsection{Statistical analysis}

Statistical analysis was performed using a $t$-test or one-way ANOVA (Bonferroni's post-test) through Graph Pad Prism 5 software. The results where $\mathrm{P}<0.05$ were considered statistically significant.

\section{Results}

\subsection{In vitro results}

3.1.1. Macrophages better control internalized amastigote growth under the effect of combined Glucantime ${ }^{\circledR}+$ QNZ therapy

The effect of QNZ, Glucantime ${ }^{\circledR}$ and the combined therapy on the inhibition of amastigote growth inside the infected macrophages was investigated. The control group (G1) was represented by macrophages infected with L. amazonensis. Fig. $1 \mathrm{~A}$ shows that all groups were able to significantly decrease the amastigote counts compared to the $\mathrm{G} 1$ infected group. The greatest inhibition profiles were observed when infected macrophages were incubated with QNZ alone and in combination with Glucantime ${ }^{\circledR}$, as can be visualized in the panels of Fig. 1B (G2 and G4 groups, respectively).

3.1.2. L. amazonensis-infected macrophages under the effect of QNZ alone and combined with Glucantime ${ }^{\circledR}$ produce high levels of NO instead of cytokines

Regarding the assay conducted to measure cytokine levels produced by the infected macrophages incubated with the drugs, Table 1 shows that cells from G2 and G4 groups produced lower levels of IL-1 $\beta$ than the control group (G1). The same phenomenon was observed when infected macrophages from those groups were assessed for IL-12. On the other hand, cells that received Glucantime ${ }^{\circledR}$ alone (G3 group) were able to increase IL-1 $\beta$ but not IL-12 production compared to G1. All groups were not able to produce detected IL-10 levels.

Fig. 2 shows the nitrite levels produced by the infected macrophages. The greatest production profiles were observed when the cells were incubated with QNZ alone (G2 group) and combined with Glucantime ${ }^{\circledR}$ (G4 group). On the other hand, the G3 group was not able to significantly increase the nitrite levels compared to G1. 
A

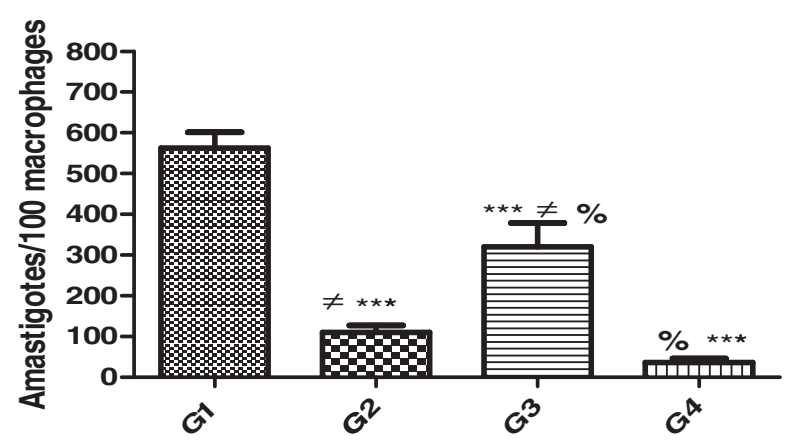

B
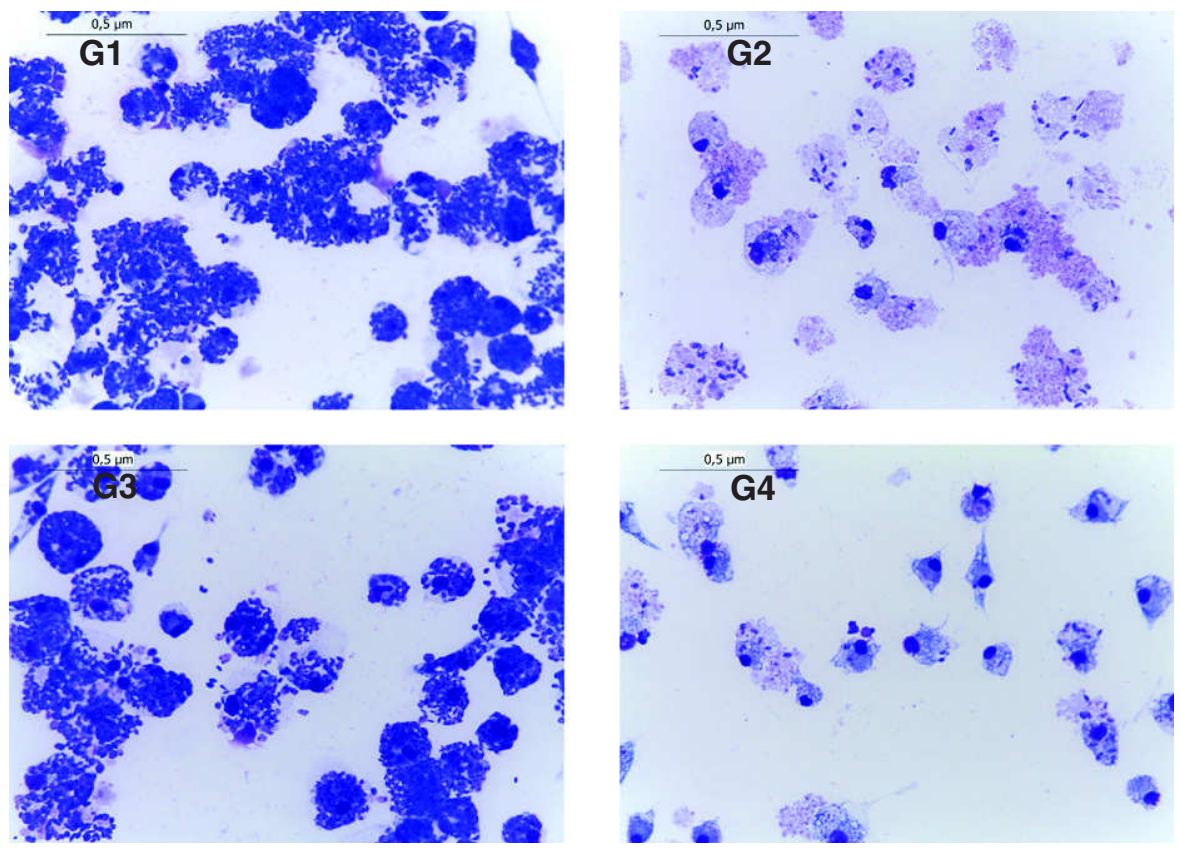

Fig. 1. Analysis of the parasites' growth into the L. amazonensis-infected macrophages. (A) Amount of amastigotes per 100 macrophages counted. Peritoneal macrophages were infected with promastigote forms in the stationary growth phase at a ratio of 5 parasites per cell (G1 group). The infected cells were incubated for $48 \mathrm{~h}$ with $10 \mu \mathrm{M}$ of QNZ (G2 group), with $300 \mu \mathrm{g} / \mathrm{mL}$ of Glucantime ${ }^{\circledR}$ (G3 group) and with the combination Glucantime ${ }^{\circledR}+$ QNZ (G4 group). Each bar represents the mean \pm S.E.M ( $\mathrm{n}=3$ ) of two independent experiments. ${ }^{* * *} \mathrm{P}<0.001$ compared to $\mathrm{G} 1 ; \neq \mathrm{P}<0.05$ compared to $\mathrm{G} 2 ; \% \mathrm{P}<0.05$ compared to G3. (B) Light micrographs (representative of triplicate) of peritoneal macrophages as described above (G1 to G4 group). Magnification $1000 \times, 0.5 \mu \mathrm{m}$ scale.

\subsection{In vivo results}

3.2.1. Combined TNF- $\alpha$ inhibition and Glucantime® therapy decreases lesion sizes of the infected paws

Fig. 3 shows the progression of the paw lesions over 8 days. Contralateral paw (CP) represents uninfected paws from evaluated mice. The average lesion size of the infected control group (G1) was very similar to QNZ-treated mice (G2) both at days 1 and 8 and the increases were statistically significant compared to $\mathrm{CP}$ group. The group treated with Glucantime ${ }^{\circledR}(\mathrm{G} 3)$ displayed decreased lesion sizes compared to G1 and G2 groups. Mice that received combined QNZ drug and Glucantime ${ }^{\circledR}$ therapy (G4) showed greater reductions of the lesion size compared to other infected groups.

Table 1

Effect of the QNZ, Glucantime® and their combination on the production of cytokines by Leishmania-infected macrophages.

\begin{tabular}{llll}
\hline Cytokines $(\mathrm{pg} / \mathrm{mL})$ & Groups & & \\
\cline { 2 - 4 } & G1 & G2 & G3 \\
\hline IL-1 & $283.83 \pm 34.55$ & $188.17 \pm 7.14^{* * *}$ & $299.11 \pm 13.89^{\# \#}$ \\
IL-10 & n.d. & n.d. & n.d. \\
IL-12 & $1180.00 \pm 110.50$ & $53.33 \pm 28.28^{* * *}$ & $1006.67 \pm 114.65^{\# \# \#}$ \\
\hline
\end{tabular}

Murine peritoneal macrophages were infected with L. amazonensis only (G1 group); infected and incubated for 48 h with QNZ (G2 group); with Glucantime $®$ (G3 group) and with the combination Glucantime ${ }^{\circledR}+$ QNZ (G4 group). Each value represents mean \pm S.E.M $(n=4)$ of two independent experiments. ${ }^{* *} \mathrm{P}<0.01$ and ${ }^{* * *} \mathrm{P}<0.001$ compared to $\mathrm{G} 1$; \#\#P $<0.01$ and \#\#\#P $<0.001$ compared to $\mathrm{G} 2 ; \% \% \mathrm{P}<0.01$ and $\% \% \mathrm{P}<0.001$ compared to $\mathrm{G} 3$. n.d. $=$ not detected. 


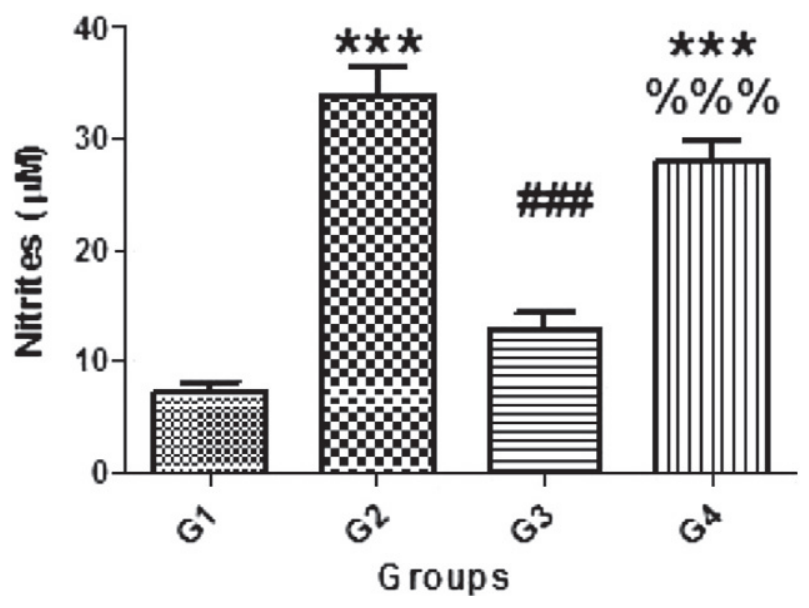

Fig. 2. Nitrite levels were quantified by Griess reaction in the supernatants of cells. L. amazonensis-infected macrophages (G1 group) were incubated for $48 \mathrm{~h}$ with $10 \mu \mathrm{M}$ of QNZ (G2 group), with $300 \mu \mathrm{g} / \mathrm{mL}$ of Glucantime ${ }^{\circledR}$ (G3 group) and with the combination Glucantime ${ }^{\circledR}+$ QNZ (G4 group). Each bar represents the mean \pm S.E.M $(n=3)$ of two independent experiments. ${ }^{* * *} \mathrm{P}<0.001$ compared to $\mathrm{G} 1$; \#\#\#P $<0.001$ compared to G2 and $\% \% \mathrm{P}<0.001$ compared to $\mathrm{G} 3$.

3.2.2. The absence of TNF- $\alpha$ during pentavalent antimonial therapy greatly reduces amastigote counts in infected paws

Histological analysis was conducted in order to observe and count the amount of amastigotes in the L. amazonensis-infected paws. Fig. 4A and $B$ shows the histological sections of the untreated infected group (G1) and the infected group treated with QNZ (G2), respectively. Both sections display the highest counts of amastigote nests in the tissue (Fig. 4E). The infected group treated with Glucantime ${ }^{\circledR}$ (G3) (Fig. 4C) presented a statistically significant decrease in the amastigote counts (Fig. 4E). In an intriguing manner, infected mice that received the combination QNZ + Glucantime ${ }^{\circledR}$ (G4) (Fig. 4D) presented the greatest reduction observed for the amount of amastigotes (Fig. 4E).

\subsubsection{QNZ + Glucantime ${ }^{\circledR}$ administration increases IL-1 $\beta$ production}

Fig. 5 shows IL-1 $\beta$ levels detected in the supernatant obtained from the excised infected paws. Infected G1, G2 and G3 groups display no statistical difference between the cytokine levels produced (ranging from 100 to $260 \mathrm{pg} / \mathrm{mL}$ ). On the other hand, IL-1 $\beta$ produced by infected mice that received combined QNZ + Glucantime ${ }^{\circledR}$ were the highest levels detected in the paw-homogenized tissue (about $500 \mathrm{pg} / \mathrm{mL}$ ).

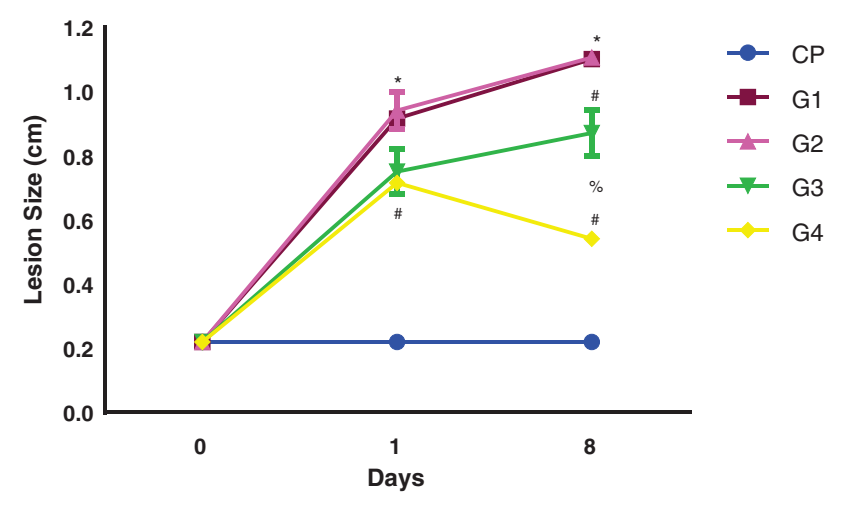

Fig. 3. Lesion sizes of infected paws during the treatments. Contralateral paw (CP) of each animal; Control infected group that received PBS $1 \times(\mathrm{G} 1)$; Group that received QNZ (G2); Group treated with Glucantime ${ }^{\circledR}$ (G3); Group treated with combined Glucantime ${ }^{\circledR}+$ QNZ QNZ administration (G4). The means \pm S.E.M of two independent experiments with statistically significant differences were represented by the symbols ${ }^{*} \mathrm{P}<0.05$ compared to $\mathrm{CP} ; \# \mathrm{P}<0.05$ compared to $\mathrm{G} 1 ; \% \mathrm{P}<0.05$ compared to $\mathrm{G} 2$.

\section{Discussion}

Several studies have shown that dermal $L$. amazonensis infection is able to activate some cellular signaling pathways, such as NF- $\kappa \mathrm{B}$, L-Arginine, inflammasome and others, triggering the production of inflammatory cytokines, microbicidal molecules and also further mechanisms of its escape from the $\mathrm{T}_{\mathrm{H}} 1$-protective response. Interestingly, Leishmania parasites possess the ability to modulate different patterns of inflammatory signaling pathways and to decrease host antimicrobial responses. As a consequence, during the infection, NF-KB activity, which normally plays a crucial role in the initiation of the innate immune response and protective $\mathrm{T}_{\mathrm{H}} 1$-mediated immunity, can be down-regulated, leading to a kind of macrophage tolerance and hyporesponsiveness [15].

All this previous information is well known in the literature, but especially for $L$. amazonensis infection, where the escape mechanisms of the parasite are evident, no data were reported about the effect of experimental therapy with pentavalent antimonials combined with TNF- $\alpha$ blockade, using a NF-KB pathway inhibitor. Since the presence of this cytokine is important for the activation of APCs, such as macrophages, by inducing IFN- $\gamma$ and NO production $[16,17]$, in this study, we investigated whether during antimonial therapy the systemic TNF$\alpha$ blockade could allow for better prognostic of the infection. An initial set of in vitro experiments was conducted to better understand the effect of the combined Glucantime ${ }^{\circledR}+$ QNZ treatment on L. amazonensisinfected macrophages. The results showed that macrophages could better control internalized amastigote growth under the effect of this combined treatment, displaying greater inhibition of amastigote counts than the control infected group (Fig. 1A and B). The findings suggest that inhibition of the NF- $\kappa B$ signaling pathway could benefit infected macrophages to increase their antimicrobial mechanisms. Interestingly, the low amastigote counts achieved inside the macrophages were not related to the increase of the protective cytokines produced from the $\mathrm{T}_{\mathrm{H}} 1$-response profile, such as IL-12 and IL-1 $\beta$. Instead, L. amazonensisinfected macrophages under the effect of QNZ alone or combined with Glucantime ${ }^{\circledR}$ were able to produce high levels of nitrites, as an indirect measurement of NO. In this context, it is known that M1-phenotype macrophages express NO-dependent leishmanicidal activity for controlling Leishmania infection. [8]. The in vitro results reached in this study suggest that decreased IL-12 and IL-1 $\beta$, without increased IL-10 levels produced by the infected macrophages under the effect of the NF- $\kappa B$ pathway inhibitor (QNZ) is related to enhanced antileishmanial activity, reflected by greater NO production.

Although some authors have described that antimonial therapy can increase proinflammatory cytokines, such as TNF- $\alpha$ and IL-1 $\beta[18,19]$, no study in leishmaniasis was conducted regarding this therapy combined with the NF- $\kappa$ B inhibitor. Some studies have been conducted in order to investigate the role of IL- $1 \beta$ in enhancing antimicrobial host defense, showing that the Nlrp3 inflammasome complex (a nucleotide-binding receptor) is activated during $L$. amazonensis infection [20]. In light of this finding, an infection model was conducted in mice and the results showed that infected mice that received the combined QNZ + Glucantime ${ }^{\circledR}$ administration were able to produce the highest IL-1 $\beta$ levels detected from the paw tissues (Fig. 5). This finding is strongly related to the observations provided by other experiments conducted, which revealed that lesion sizes of the infected paws (Fig. 3 ) and also their amastigote counts (Fig. 4) were significantly reduced. Interestingly, increased IL- $1 \beta$ levels obtained from the paw tissues were not reproducible compared to those from the in vitro assay conducted with isolated infected macrophages. It can be speculated that TNF- $\alpha$ blockade employing QNZ was not enough to display the same phenomenon as in the in vivo context, where the treatment was done for five days and also the immunological system was present. The results suggest that other cells besides macrophages, such as dendritic cells, could be activated by the therapy and also produced IL-1ß. 


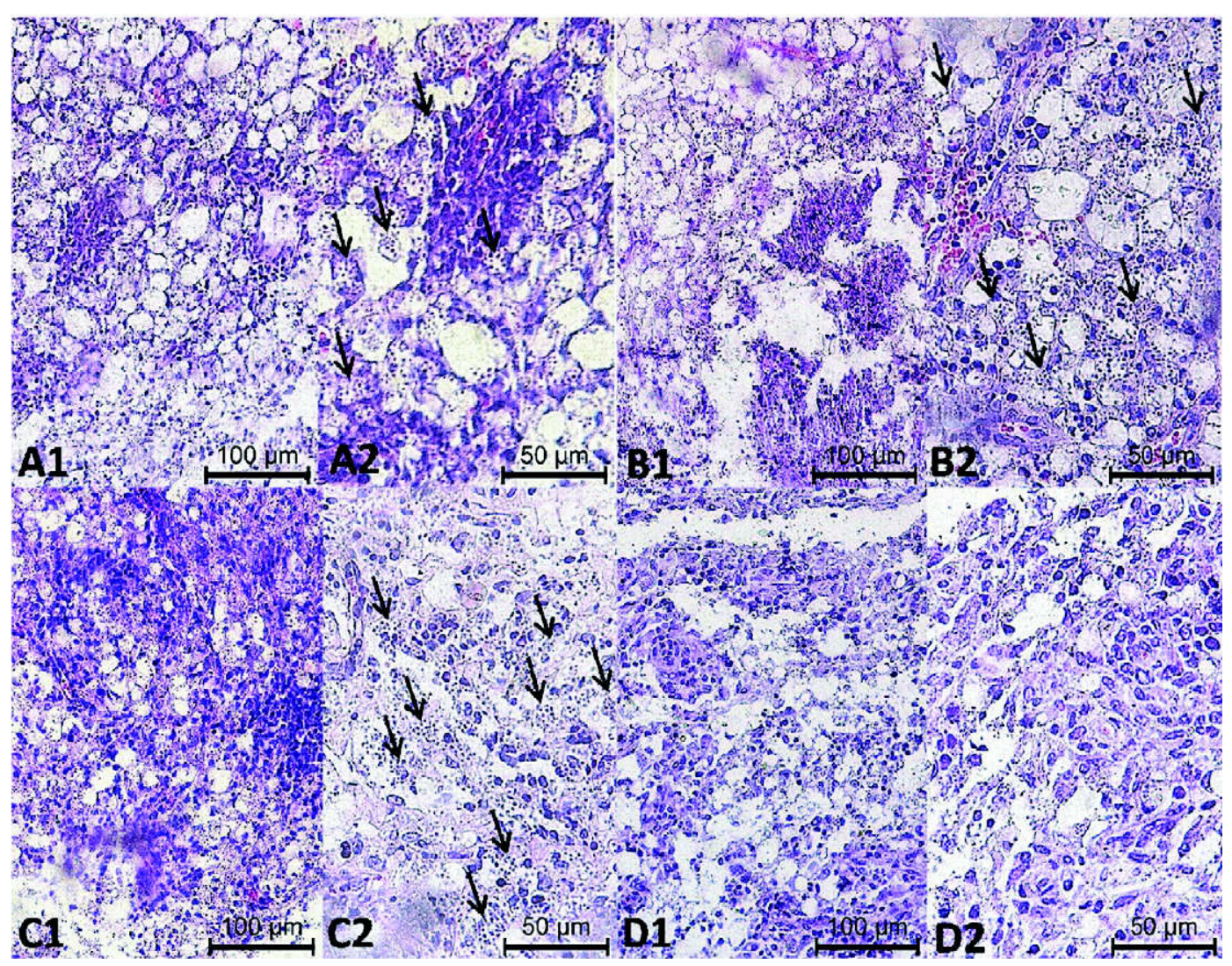

\section{$\mathbf{E}$}

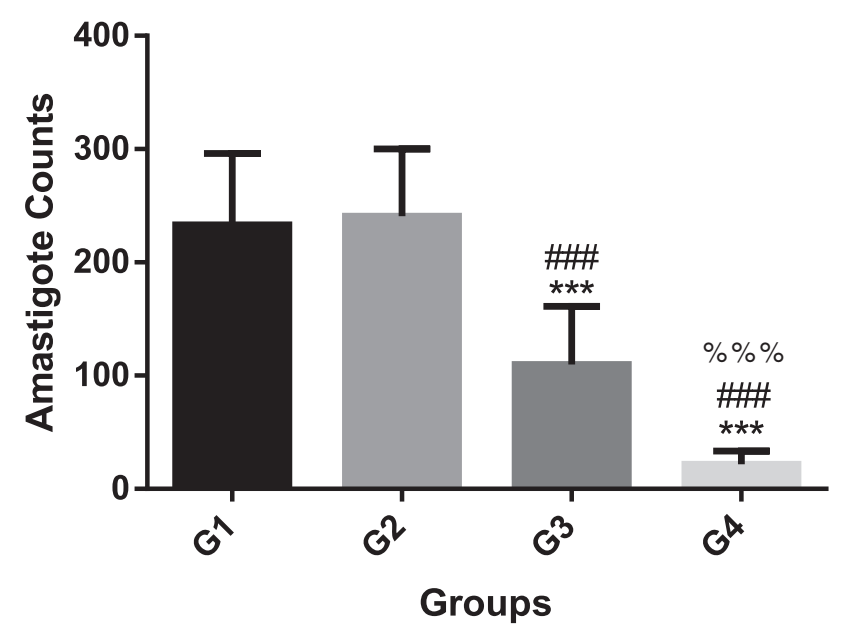

Fig. 4. Representative histological sections from paws tissue stained with HE. Panel (A) represents control infected untreated group; (B) represents group that received QNZ; (C) represents mice treated with Glucantime ${ }^{8}$; (D) represents infected group that received combined Glucantime ${ }^{\circledR}+$ QNZ. The amastigote forms are indicate by black arrows. Magnifications of each panel at 200 and $400 \times$ (100 and $50 \mu \mathrm{m}$ scale, respectively). (E) Amastigote forms of each group were counted in 5 to 6 fields using ImageJ software (NIH) from 4 different sections (mean \pm S.E.M of two independent experiments).

As described in the literature, proinflammatory cytokines, such as TNF- $\alpha$ and IL-1 $\beta$ can be increased during antimonial therapy in leishmaniasis [19,21]. However, with the results achieved in this study, we can speculate and suggest that besides the role of these important cytokines for the maintenance of host defense mechanisms against Leishmania infection, precise regulation of their levels in order to reach a better prognosis during the infection is extremely important. In this regard, the administration of QNZ was able to maintain low levels of TNF- $\alpha$ (about $100 \mathrm{pg} / \mathrm{mL}$ ) produced in local infected paws. We could also observe that infected mice treated with Glucantime ${ }^{\circledR}$ presented the highest levels of this cytokine (about $600 \mathrm{pg} / \mathrm{mL}$ ) and under the combined QNZ + Glucantime ${ }^{\circledR}$ administration about $250 \mathrm{pg} / \mathrm{mL}$ of TNF- $\alpha$ were detected (data not shown).

Taken together, our results show that antimonial therapy when combined with the NF- $\kappa B$ inhibitor, which is able to block TNF- $\alpha$ production systemically, is a promising pharmacological approach against experimental leishmaniasis. One of the possible mechanisms involved could be the proinflammatory cytokine balance with high 


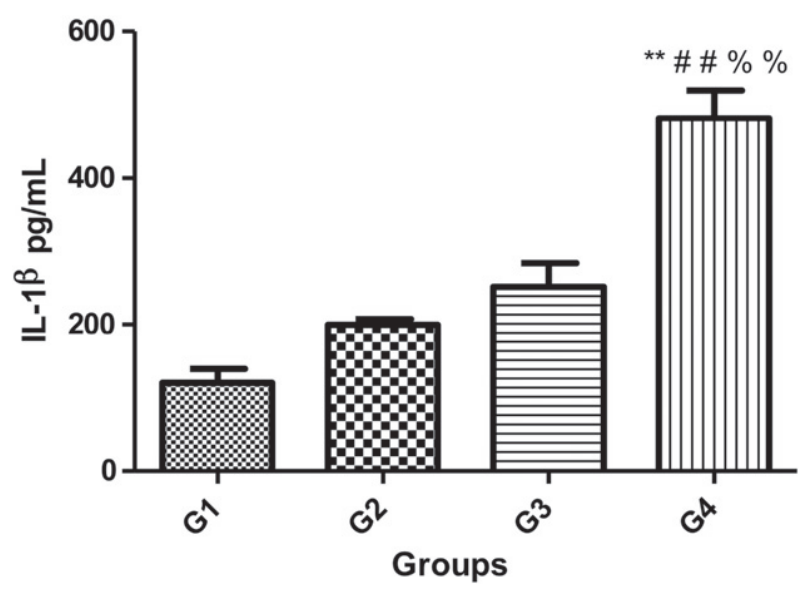

Fig. 5. Determination of IL-1 $\beta$ levels in the homogenized paw-infected tissue. G1 (control infected group, untreated); G2 (group that received QNZ); G3 (group treated with Glucantime ${ }^{\circledR}$ ); G4 (group that received combined Glucantime ${ }^{\circledR}+$ QNZ). Means \pm S.E.M of two independent experiments with statistically significant differences were represented by symbols ${ }^{* *} \mathrm{P}<0.01$, compared to control infected group; \#\#P $<0.01 \mathrm{com}$ pared to the group treated with the inhibitor and $\mathrm{QNZ} \% \% \mathrm{P}<0.01$ compared to the group treated with Glucantime ${ }^{\circledR}$.

NO production by macrophages during amastigotes' survival inside the macrophages. It is also important to point out that one of the features of NF-kB is its role during the crosstalk event with other signal transduction pathways, such as MAPK/ERK signaling cascades. The high IL-1 $\beta$ levels produced by macrophages and other APCs in infected paws during QNZ + Glucantime ${ }^{\circledR}$ administration were able to maintain a better cellular activation profile with increased NO production, avoiding TNF- $\alpha$ overproduction, decreasing Leishmania replication and enhancing healing in the infected paws. Further studies will be necessary to extend the understanding about the cellular mechanisms developed by the host to solve the infection and more importantly, how the balance of cytokines and other immune mediators produced by leukocytes interacts with the current antimonial therapy.

\section{Acknowledgments}

The authors are grateful to Ms. Amy Nicole Grabner for the language revision and Felipe Stegun for the technical support provided with histological sections. This work was financially supported by Fundação Oswaldo Cruz (Encomenda Papes, proc. 407540/2012-1), Conselho Nacional de Desenvolvimento Científico e Tecnológico (CNPq) and Coordenação de Aperfeiçoamento de Nível Superior (CAPES), from Brazil.

\section{References}

11] O.L.S. Almeida, J.B. Santos, Advances in the treatment of cutaneous leishmaniasis in the new world in the last ten years: a systematic literature review, An Bras Dermatol 86 (2011) 497-506

[2] J. Alexander, D.G. Russell, The interaction of Leishmania species with macrophages, Adv. Parasitol. 31 (1992) 175-254.

[3] M.K. Basu, M. Ray, Macrophage and Leishmania: an unacceptable coexistence, Crit. Rev. Microbiol. 31 (2005) 145-154.

[4] B.J. Nelson, P. Ralph, S.J. Green, C.A. Nacy, Differential susceptibility of activated macrophage cytotoxic effector reactions to the suppressive effects of transforming growth factor-beta 1, J. Immunol. 146 (1991) 1849-1857.

[5] F.Q Cunha, S. Moncada, F.Y. Liew, Interleukin-10 (IL-10) inhibits the induction of nitric oxide synthase by interferon-gamma in murine macrophages, Biochem. Biophys. Res. Commun. 182 (1992) 1155-1159.

[6] R.T. Gazzinelli, I.P. Oswald, S. Hieny, S.L. James, A. Sher, The microbicidal activity of interferon-gamma-treated macrophages against Trypanosoma cruzi involves an L-arginine-dependent, nitrogen oxide-mediated mechanism inhibitable by interleukin-10 and transforming growth factor-beta, Eur. J. Immunol. 22 (1992) 2501-2506.

[7] S.J. Green, C.A. Nacy, M.S. Meltzer, Cytokine-induced synthesis of nitrogen oxides in macrophages: a protective host response to Leishmania and other intracellular pathogens, J. Leukoc. Biol. 50 (1991) 93-103.

[8] D.M. Mosser, J.P. Edwards, Exploring the full spectrum of macrophage activation, Nat. Rev. Immunol. 8 (2008) 958-969.

[9] L.C. Afonso, P. Scott, Immune responses associated with susceptibility of C57BL/10 mice to Leishmania amazonensis, Infect. Immun. 61 (1993) 2952-2959.

[10] S. Nylén, L. Eidsmo, Tissue damage and immunity in cutaneous leishmaniasis, Parasite Immunol. 34 (2012) 551-561.

[11] D.C. Soares, T.C. Calegari-Silva, U.G. Lopes, V.L. Teixeira, I.C.N. de Palmer Paixão, C. Cirne-Santos, et al., Dolabelladienetriol, a compound from Dictyota pfaffii algae, inhibits the infection by Leishmania amazonensis, PLoS Negl. Trop. Dis. 6 (2012) e1787.

[12] H.A. Lessa, P. Machado, F. Lima, A.A. Cruz, O. Bacellar, J. Guerreiro, et al., Successful treatment of refractory mucosal leishmaniasis with pentoxifylline plus antimony, Am. J. Trop. Med. Hyg. 65 (2001) 87-89.

[13] W.N. Oliveira, L.E. Ribeiro, A. Schrieffer, P. Machado, E.M. Carvalho, O. Bacellar, The role of inflammatory and anti-inflammatory cytokines in the pathogenesis of human tegumentary leishmaniasis, Cytokine 66 (2014) 127-132.

[14] N.B. Barros, V. Migliaccio, V.A. Facundo, P. Ciancaglini, R.G. Stábeli, R. Nicolete, et al., Liposomal-lupane system as alternative chemotherapy against cutaneous leishmaniasis: macrophage as target cell, Exp. Parasitol. 135 (2013) 337-343.

[15] K. Reinhard, M. Huber, M. Lohoff, A. Visekruna, The role of NF-kB activation during protection against Leishmania infection, Int. J. Med. Microbiol. 302 (2012) 230-235.

[16] R.M. Palmer, D.S. Ashton, S. Moncada, Vascular endothelial cells synthesize nitric oxide from L-arginine, Nature 333 (1988) 664-666.

[17] I. Sakuma, D.J. Stuehr, S.S. Gross, C. Nathan, R. Levi, Identification of arginine as a precursor of endothelium-derived relaxing factor, Proc. Natl. Acad. Sci. U. S. A. 85 (1988) 8664-8667.

[18] A. Kocyigit, S. Gur, M.S. Gurel, V. Bulut, M. Ulukanligil, Antimonial therapy induces circulating proinflammatory cytokines in patients with cutaneous Leishmaniasis, Infect. Immun. 70 (2002) 6589-6591.

[19] B.J. Berger, A.H. Fairlamb, Interactions between immunity and chemotherapy in the treatment of the trypanosomiases and leishmaniases, Parasitology 105 (Suppl.) (1992) S71-S78.

[20] D.S. Lima-Junior, D.L. Costa, V. Carregaro, L.D. Cunha, A.L.N. Silva, T.W.P. Mineo, et al., Inflammasome-derived IL-1 $\beta$ production induces nitric oxide-mediated resistance to Leishmania, Nat. Med. 19 (2013) 909-915.

[21] R.R. De Saldanha, M.C. Martins-Papa, R.N.R. Sampaio, M.I. Muniz-Junqueira, Meglumine antimonate treatment enhances phagocytosis and TNF- $\alpha$ production by monocytes in human cutaneous leishmaniasis, Trans. R. Soc. Trop. Med. Hyg. 106 (2012) 596-603. 